ARTICULO DE INVESTIGACIÓN

\title{
Caracterización de hojas de mazorca de maíz y de bagazo de caña para la elaboración de una pulpa celulósica mixta
}

\section{Characterization of maize leaves and of sugarcane bagasse to elaborate of a mixed cellulose pulp}

\author{
Maribel Prado-Martínez¹, José Anzaldo-Hernández², \\ Bruno Becerra-Aguilar2, Hilda Palacios-Juárez², \\ José de Jesús Vargas-Radillo² y Maite Rentería-Urquiza1
}

\begin{abstract}
RESUMEN
En este artículo se presentan los resultados obtenidos en la caracterización química y morfológica de las hojas de mazorca de maíz (Zea mays) y del bagazo de caña (Saccharum officinarum L.). Se analizaron porcentajes de $\alpha$-celulosa, holocelulosa, lignina y cenizas, según las Normas Tappi para cada componente y el método de Jayme-Wise. Se determinaron las propiedades biométricas de las fibras de cada planta (longitud y diámetro, grosor de la pared y diámetro del lumen). Además, se estimaron los índices de calidad de pulpa para papel. Este estudio se hizo con la finalidad de tener información de ambas plantas para su posterior tratamiento de pulpeo, blanqueo y mezcla, para la obtención de una pulpa celulósica óptima, a partir de sus residuos. Los resultados indican que el contenido de holocelulosa en las hojas de mazorca se encuentra en el intervalo de los materiales usados comúnmente en la producción de pulpa para papel y es cercano al observado en el bagazo de caña. La caracterización morfológica de las fibras mostró que la longitud de las mismas es muy parecida, no así el ancho, el espesor y el lumen. Las hojas de mazorca de maíz presentaron fibra con mejores características para la fabricación de una pulpa óptima, al situarse en el intervalo de "muy buena", según la clasificación de Runkel. Por su parte, la fibra de bagazo de caña, presentó características "buenas".
\end{abstract}

PALABRAS CLAVE:

Caracterización química, morfología de fibra, pulpa celulósica, Saccharum officinarum, Zea mays.

\begin{abstract}
This article presents the results obtained in chemical and morphological, characterization of corn cob leaves (Zea mays), and bagasse of sugarcane (Saccharum officinarum). Percentage of $\alpha$-cellulose, holocellulose, lignin and ashes, according to the Tappi standards and the Jayme-Wise method for each component, were analyzad. Biometric properties of each plant fibers (length and diameter, wall thickness and lumen diameter) were determined. Indices of quality of paper pulp for paper were also estimated. This study was conducted in order to get information of both plants for further treatment of pulping, bleaching and mixing, to obtain an optimal cellulosic pulp, from their waste. The results indicate that the content of holocellulose in the leaves of maize ear is in the range of materials commonly used in the production of pulp for paper and is close to that observed in sugarcane bagasse.
\end{abstract}

1 Departamento de Química, CUCEI-Universidad de Guadalajara. Blvd. Marcelino García Barragán 1451, C.P. 44430, Guadalajara Jalisco, México. C.e.: reurmaite@yahoo.es

2 Departamento de Madera, Celulosa y Papel, CUCEI-Universidad de Guadalajara. Carretera GuadalajaraNogales, Km. 141/2 Predio Las Agujas, Nextipac, Jalisco, México. 
Morphological characterization of fibers showed that the length of them is very similar, unlike the width, thickness and lumen diameter. Corn cob leaves presented fiber with best features for an optimal pulp manufacturing. They were "very good", according to Runkel's classification. For its part, sugarcane bagasse fiber, presented "good" features.

KEY WORDS:

Chemical characterization, morphology of fiber, cellulose pulp, Saccharum officinarum, Zea mays.

\section{INTRODUCCIÓN}

Teniendo en cuenta que el ciclo del papel inicia en el bosque, es de vital importancia para las industrias de la celulosa y del papel, impulsar en la medida de lo posible el desarrollo forestal y la utilización racional de los recursos forestales. Es necesario complementar lo anterior con una cultura del reciclaje, con la utilización de los residuos generados con otras fibras utilizadas en la fabricación de celulosa y la producción sustentable del papel; todo ello en la búsqueda de un desarrollo con calidad y beneficios ambientales, económicos y sociales de trascendencia para las generaciones actuales y las futuras. (Conafor, 1992; Prado, 2007).

De acuerdo con la Organización de la Naciones Unidas para la Agricultura y la Alimentación (FAO), los productos forestales no madereros (PFNM) son bienes de origen biológico, distintos de la madera, derivados del bosque, de otras áreas forestales y de los árboles fuera de los bosques. Los PFNM pueden recolectarse en forma silvestre o producirse en plantaciones forestales o sistemas agroforestales. Estos productos, en su gran mayoría de origen vegetal, son utilizados como alimentos y aditivos alimentarios (semillas comestibles, hongos, frutos, condimentos, aromatizantes), fibras, instrumentos o utensilios, resinas, gomas, y otros usados con fines medicinales, cosméticos o culturales (López, 2008).

México dispone de considerables reservas de material para la producción de celulosa a partir de fibras no maderables, tales como resinas, fibras, gomas, ceras, rizomas y otros (hongos, semillas, hojas, nopal, tallos, frutos, musgo, heno, etc.) (Semarnat, 2011; Tapia, 2008). La celulosa puede obtenerse de la planta virgen, pero también de los residuos generados por ellas. De esta forma se aprovecharía el excedente de los desechos producidos, se crearía un material con buenas aplicaciones y surgiría un nuevo mercado industrial (Semarnat, 2011; Tapia, 2008).

En el año 2011, la producción forestal no maderable en México fue de 63,9 miles de toneladas. Esta cifra es tan solo inferior en $3,8 \%$, respecto a la producción del año anterior. La producción más alta en los últimos diez años se sitúa en 2003 con 97,6 miles de toneladas (Semarnat, 2011; Saval, 2012; Casey, 1990).

Con una combinación adecuada de fibras vegetales no maderables es posible producir pulpa celulósica de calidad. Entre las ventajas que presentan frente a las fibras maderables están un menor consumo de reactivos y de energía, además de producir menos residuos tóxicos durante su tratamiento (López, 2008).

El interés en utilizar los materiales fibrosos no maderables como los residuos agrícolas es múltiple:

1. Sustituir, por lo menos parcialmente, la materia prima maderable para la fabricación de papel, disminuyendo por lo tanto la deforestación que da lugar a problemas ambientales graves.

2. Disponer de residuos agrícolas, ya que la industria agrícola cuenta con grandes excedentes de las cosechas. 
3. Con el empleo de estas materias primas se podrían aumentar las producciones de pulpas y derivados, consiguiendo abastecer el consumo de fibras en México.

Pero es necesaria la caracterización de las fibras para conocer su composición química, su morfología y la cantidad que se genera. Con esto se pueden definir las tecnologías más apropiadas para su aprovechamiento y posterior tratamiento, aunado a un enfoque de responsabilidad ambiental.

El maíz es una planta herbácea anual, nativa del hemisferio norte, originaria de México desde hace unos $10 \mathrm{mil}$ años y que se cultiva en todo el mundo. Representa una de las principales fuentes de alimentación para casi todas las comunidades de México. Las regiones ganaderas de Jalisco, Guanajuato, Querétaro, Michoacán, Aguascalientes, Hidalgo, la región lagunera y otras, cada año destinan más maíz para la alimentación animal, ya sea para ensilaje o como forraje seco. Solo en Jalisco hay un estimado de 100,000 hectáreas dedicadas a usos forrajeros (Ron et al., 2006). La producción de biomasa residual que genera un cultivo de maíz (cañas, hojas de maíz, hojas de mazorca de maíz y mazorcas) fluctúa entre las 20 y 35 toneladas por hectárea y en el maíz de choclo (cañas y hojas), varía de 16 a 25 toneladas por hectárea (González, 1995; IDEA, 2007; Imba, 2011).

La caña de azúcar (Saccharum officinarum L.) es una gramínea tropical, un pasto gigante emparentado con el sorgo y el maíz. Procede del Extremo Oriente, de donde llegó a España en el siglo IX. En México apareció tras la Conquista, instalándose las primeras industrias azucareras en las partes cálidas del país, como parte de la colonización. Suministra $70 \%$ de la demanda internacional de azúcar, seguida de la remolacha. El azúcar se obtiene del jugo fresco y dulce de la caña, sus hojas y tallos se utilizan como forraje para el ganado. Hay diferentes tipos de azúcar, desde el piloncillo o panela hasta el azúcar refinado, los cuales se usan como alimento básico del hombre o como materia prima para la industria. Ésta lo transforma en alcohol etílico, ácido láctico, dextrosa y glicerina. (Rutiaga et al., 2002; Sidney, 1991; Matta, 1991). Es la principal actividad económica en 15 estados y 227 municipios de la república, donde se siembra y procesa caña de azúcar, aunque tiene presencia en 667 municipios (Aguilar et al., 2011). Los estados con mayor producción son Veracruz, seguido de Jalisco, donde se alcanzan las 6 toneladas anuales y Oaxaca (Sagarpa, 2010). Actualmente, y debido a los problemas medioambientales, se hace referencia a la caña de azúcar como fuente de biomasa para obtener energía renovable. Más de $70 \%$ de la producción de los residuos de la industria azucarera se destinan a la producción de bioetanol. El uso de la biomasa como energía representa en México $8 \%$ de la demanda de energía primaria y está centrada en el uso de leña residencial y de pequeñas industrias y en el uso de bagazo de caña en ingenios azucareros. Sin embargo, la bioenergía tiene un potencial mucho más amplio y podría convertirse en uno de los pilares del desarrollo sustentable del país. (Conafor, 2012).

Dado que las propiedades mecánicas del papel dependen no sólo de las propiedades morfológicas, sino también del contenido de lignina y celulosa, es importante conocer la proporción de estos en la materia prima fibrosa. Asimismo, la composición química indica el porcentaje de rendimiento teórico a obtener durante el proceso de transformación a pulpa, además de la cantidad de agentes químicos para su tratamiento. Además, debido a la gran cantidad de residuos que la industria agraria del maíz (aproximada- 
mente 10 toneladas de residuos por hectárea) (Sener, 2011) y los ingenios azucareros (por cada tonelada de caña procesada se obtienen de $30 \mathrm{~kg}$ a $50 \mathrm{~kg}$ de este residuo) (Pérez et al., 2011) generan en México, se tendría una solución óptima para el problema del manejo y tratamiento de los mismos, así como para el aprovechamiento de los recursos naturales. Esto es importante puesto que estamos en una época crucial en el desarrollo futuro de la humanidad y dentro de un contexto integral sobre protección ambiental y sustentabilidad.

\section{OBJETIVOS}

Determinar los componentes químicos de dos plantas no madereras, maíz (hojas de mazorca) (Zea mays) y bagazo de caña (Saccharum officinarum) y conocer la morfología de sus fibras, con el fin de evaluar la factibilidad de su uso en la producción de pulpa celulósica mixta.

\section{METODOLOGÍA}

Las hojas de maíz utilizadas fueron proporcionadas por la fábrica tamalera "La Candelaria" de la ciudad de Guadalajara. El bagazo de caña fue recolectado de la zafra de 2006 en el ingenio "José María Martínez" del municipio de Tala, Jalisco. Tanto el muestreo como la preparación de las muestras para los análisis se llevaron a cabo de acuerdo con lo descrito en las normas Tappi (Tappi, 1997) y en el ensayo Jayme-Wise.

\section{Preparación de la muestra para caracterización química y morfológica (T 264 cm-97)}

El material se pasó por un molino de martillo y se tamizó en un equipo Ro-Tap. El primer tamiz fue de malla número 40
$(0,420 \mathrm{~mm})$ y el segundo de malla número $60(0,25 \mathrm{~mm})$. La muestra retenida en este último tamiz fue la considerada para la caracterización química. Se tomaron entre $9 \mathrm{~g}$ y $10 \mathrm{~g}$ de muestra, peso base seca, para los análisis químicos. De acuerdo con la norma T 264 cm-97, la muestra debe estar libre de extraíbles, por lo cual se llevó a cabo un proceso de extracción Soxhlet usando $200 \mathrm{ml}$ de solvente, etanol: tolueno (1:2 por volumen) durante 6 horas, seguido de un lavado con etanol y una segunda extracción también con etanol (95\% pureza), durante 4 horas. Después, la muestra fue lavada con $500 \mathrm{ml}$ de agua destilada y secada a temperatura ambiente.

\section{Determinación de los componentes químicos}

1. Determinación de holocelulosa (método Jayme-Wise) (Wise et al., 1946)

Para la cuantificación de la holocelulosa se pesaron $2,5 \mathrm{~g}$ de muestra libre de extraíbles en un matraz Erlenmeyer de $250 \mathrm{ml}$ de capacidad, aplicando un tratamiento de cinco etapas con clorito sódico $\left(\mathrm{NaClO}_{2}\right)$ en medio ácido $\left(\mathrm{CH}_{3} \mathrm{COOH}\right)$. Cada etapa duró 1 hora y se realizó a una temperatura de $70{ }^{\circ} \mathrm{C}$ en baño María con agitación ocasional. Después se enfrío la muestra, colocando el Erlenmeyer en un recipiente con hielo, hasta que la temperatura fue de $10{ }^{\circ} \mathrm{C}$. Se filtró la muestra por un filtro Gooch de porosidad fina, se lavó con agua fría destilada, posteriormente con acetona y se dejó secar en una estufa de vacío a $105^{\circ} \mathrm{C}$ durante 24 horas. Por último, se colocó en un desecador y se pesó.

\section{Determinación de $\alpha$-celulosa}

La determinación de a-celulosa, realizada mediante la norma T 203 cm-99, es un procedimiento que se deriva 
del análisis de holocelulosa. Se pesaron 2,0 $\mathrm{g}$ de muestra de holocelulosa seca en un vaso de precipitado de $300 \mathrm{ml}$ y se añadieron $35 \mathrm{ml}$ de una solución de $\mathrm{NaOH}$ al $17,5 \%$, mezclando con una varilla de vidrio. Cada 5 minutos se añadió un volumen de 2,5 ml de la misma solución de $\mathrm{NaOH}$, hasta completar un volumen de $100 \mathrm{ml}$. Finalmente se adicionaron $100 \mathrm{ml}$ de agua destilada y se mantuvo la mezcla a $20{ }^{\circ} \mathrm{C}$ durante una hora, antes de filtrarse.

En un matraz de $250 \mathrm{ml}$ se agregaron $10 \mathrm{ml}$ de la solución filtrada más $20 \mathrm{ml}$ de $\mathrm{K}_{2} \mathrm{Cr}_{2} \mathrm{O}_{7} 0.5 \mathrm{~N}$ y $30 \mathrm{ml}$ de $\mathrm{H}_{2} \mathrm{SO}_{4}$ concentrado, dejando que la solución permaneciera caliente durante 15 minutos, tras lo cual se añadieron $50 \mathrm{ml} \mathrm{de} \mathrm{H}_{2} \mathrm{O}$ y se dejó enfriar a temperatura ambiente, añadiendo de 2 a 4 gotas de indicador "Ferroín". Se tituló con solución de $\mathrm{Fe}\left(\mathrm{NH}_{4}\right)_{2}\left(\mathrm{SO}_{4}\right)_{2} 0.1 \mathrm{~N}$ hasta color púrpura (vire; verde $\rightarrow$ púrpura). El blanco para esta titulación se preparó sustituyendo el filtrado de la pulpa con $12,5 \mathrm{ml}$ de $\mathrm{NaOH}$ a $17,5 \%$ y $12,5 \mathrm{ml}$ de agua (Vire naranjaverde-púrpura). El contenido de a-celulosa se calcula con la ecuación 1.

$$
\% \text { alfa celulosa }=100-\left[\frac{6,85\left(V_{2}-V_{1}\right) \cdot N \cdot 20}{A \cdot W}\right]
$$

donde:

$V_{1}$ : Titulación del filtrado de la pulpa, $\mathrm{ml}$

$V_{2}$ : Titulación en blanco, $\mathrm{ml}$

$N$ : Normalidad exacta de la solución de sulfato ferroso amoniacal

A: Volumen del filtrado de la pulpa usado en la oxidación, $\mathrm{ml}$

$W$ : Peso del espécimen de la pulpa secada en la estufa, $g$

\section{Determinación de lignina}

Para la determinación de lignina se aplicó la norma T 222 om-98. Se añadieron 2,0 $\mathrm{g}$ de muestra anhidra libre de extraíbles, en un vaso de precipitado de $100 \mathrm{ml}$ y se le agregaron $15 \mathrm{ml}$ de
$\mathrm{H}_{2} \mathrm{SO}_{4}$ al $72 \%$, macerando el material con una varilla de vidrio. Después se transfirió a un matraz bola agregando $400 \mathrm{ml}$ de agua destilada, se agitó suavemente y se diluyó con agua hasta alcanzar una concentración de ácido al $3 \%$ equivalente a un volumen de $575 \mathrm{ml}$. Posteriormente, se hirvió a reflujo durante 4 horas para asegurar un volumen constante de agua. La solución se decantó, manteniendo el matraz en una posición inclinada hasta que el material estuvo finalmente separado. Se filtró a través de un filtro Gooch de porosidad fina y fue lavado con agua caliente y secado a $105^{\circ} \mathrm{C} \pm 3{ }^{\circ} \mathrm{C}$ hasta alcanzar un peso constante. El cálculo se realizó con la ecuación 2 .

$$
\% \text { Lignina }=\frac{A \cdot 100}{W}
$$

donde:

A: peso de lignina, $\mathrm{g}$

$W$ : peso del espécimen secado en la estufa, $\mathrm{g}$

\section{Determinación de cenizas}

Se usó la norma T 211 om-93. Se tomaron $4 \mathrm{~g}$ de muestra libre de extractos, colocándose en un crisol de peso conocido y dejándose secar hasta peso constante. Después se quemó directamente a la llama y posteriormente se introdujo en una mufla a $500{ }^{\circ} \mathrm{C} \pm$ $15^{\circ} \mathrm{C}$, para la combustión total del carbón generado. Finamente, se dejó enfriar la muestra en un desecador y se pesó. El porcentaje de cenizas se calculó con base en el peso anhidro de la muestra (Ec. 3).

$$
\text { Ceniza }(\%)=\frac{(A \cdot 100)}{B}
$$

donde:

A: $\quad$ Peso de la ceniza, $g$

$B$ : Peso base seca del espécimen de prueba, $\mathrm{g}$ 


\section{Caracterización morfológica}

En el estudio morfológico se partió de la aplicación del método de maceración Franklin con la finalidad de obtener células aisladas. El material fibroso se colocó en tubos de ensayo añadiéndoles partes iguales de ácido acético $\left(\mathrm{CH}_{3} \mathrm{COOH}\right)$ y peróxido de hidrógeno $\left(\mathrm{H}_{2} \mathrm{O}_{2}\right)$. Se colocaron en baño María a $50^{\circ} \mathrm{C}$, hasta obtener un color blanquecino $(48 \mathrm{~h})$. El material tratado se lavó con agua destilada y después se agitó para lograr la disociación de los elementos. Las células se tiñeron ligeramente con safranina acuosa (solución acuosa colorante), lavándose e introduciéndose después en fenol glicerina $(20 \mathrm{ml}$ de fenol derretido, $40 \mathrm{ml}$ de glicerina y $40 \mathrm{ml}$ de agua). Posteriormente, se colocó el material sobre láminas, se dispersó con agua y se colocó sobre una placa de calentamiento a $60^{\circ} \mathrm{C}$ para evaporar el agua. Se tapó con un cubreobjetos y se montaron las láminas en un microscopio de proyección marca Leitz tipo 31.047.500. Se realizaron 30 mediciones de la longitud $(\mathrm{mm})$ y diámetro $(\mu \mathrm{m})$ de las fibras y diámetro de lumen $(\mu \mathrm{m})$. Cada fibra se midió en tres segmentos y al final se sumaron y se multiplicaron por un factor de 0,01014 para longitud y 0,00106 para ancho y lumen de la fibra. Con los datos obtenidos se calculó el espesor de pared (Ec. 4) e índices de calidad como coeficiente de flexibilidad (Ec. 5), relación Runkel (E. 6) y fracción de pared (Palacios, 2003; Juárez, 1993; Monteoliva, 2005). El índice de flexibilidad y la relación Runkel, se calcularon con los valores promedio medidos en el corte transversal.

$$
\begin{aligned}
\text { Espesor de pared } & =\frac{\text { Ancho fibra }- \text { Lumen }}{\text { Lumen }} \\
\text { Flexibilidad } & =\frac{\text { Lumen }}{\text { Ancho fibra }} \\
\text { Relación Runkel } & =\frac{2 \cdot \text { espesor de pared }}{\text { Lumen }}
\end{aligned}
$$

\section{RESULTADOS Y DISCUSIÓN}

\section{Caracterización química}

Los resultados obtenidos de la caracterización química de ambas plantas, se presentan en la tabla 1. Comparados con los valores encontrados en la literatura para el bagazo de caña de azúcar, se concluye, que estos dos materiales fibrosos son muy similares (Akbari, 2012; Hurter, 2001; Vega et al., 2008).

El contenido de holocelulosa en las hojas de mazorca es adecuado, dado que

Tabla 1. Composición química de la hoja de mazorca de maíz y del bagazo de caña. Comparación con valores publicados.

\begin{tabular}{lccc}
\hline Compuesto & $\begin{array}{c}\text { Hoja de mazorca } \\
\text { porcentaje (\%) } \\
\text { base seca }\end{array}$ & $\begin{array}{c}\text { Bagazo de caña } \\
\text { porcentaje }(\%) \\
\text { base seca }\end{array}$ & $\begin{array}{c}\text { Bagazo de caña de azúcar } \\
\text { porcentaje }(\%)^{*} \\
\text { base seca }\end{array}$ \\
\hline Holocelulosa & 78,86 & 73,24 & $59-76$ \\
a-Celulosa & 43,14 & 41,67 & $32-44$ \\
Lignina & 23,00 & 19,98 & $19-24$ \\
Cenizas & 0,761 & 1,300 & $1,5-5,0$ \\
\hline
\end{tabular}

* Datos obtenidos por Hurter (2001) 
se encuentra en el intervalo de los materiales usados comúnmente en la producción de pulpa para papel (67\%-70\% material base seca) (Hurter, 2001), lo mismo ocurre con el bagazo de caña. En cuanto al contenido de lignina en las hojas de mazorca, es relativamente bajo, comparado con el contenido de lignina en las maderas latifoliadas, en donde se sitúa entre $23 \%$ y $30 \%$ (Hurter, 2001). Característica más marcada para el bagazo, donde el porcentaje no supera $20 \%$. Esto indica que la carga de agentes químicos y tiempo de reacción durante el proceso de pulpeo, en ambos casos, serán inferiores o iguales a los aplicados para maderas latifoliadas y plantas anuales.

\section{Caracterización morfológica de las fibras}

\section{Propiedades biométricas de las fibras}

En la figura 1 se indican las diferentes partes de las fibras. En la tabla 2 se aprecian los resultados de las mediciones biométricas de las fibras que forman parte de las hojas de mazorca y las fibras del bagazo de caña. Dichos valores son comparados con las dimensiones de otras especies de coníferas como la Jacaranda acutifolia o la Tilia mexicana, entre otras (Tamarit, 1996), utilizadas en la industria de pulpa y papel.

Los resultados para el maíz, coinciden con lo explicado por Palacios (2003), quien propuso una clasificación y medidas en lo que respecta a la longitud promedio de las fibras de las hojas de mazorca, colocando a éstas dentro del grupo de fibras moderadamente largas. Esa longitud es incluso similar a la que presentan algunas maderas coníferas como las ya mencionadas: Jacaranda acutifolia, Tilia mexicana, Bellotia mexicana o Clethra mexicana, entre otras, y que puede alcanzar los $3 \mathrm{~mm}$ y mayor, que para otras especies de fibras no maderables como el bagazo de caña (cuyo dato también se analizó), el Kenaf, el bambú y maderas latifoliadas como el Eucalyptus globulus, donde las fibras son en general "fibras cortas" (Tabla 2) (Tamarit, 1996; Sanjuan, 1997; Estrada, 2010; Cea, 2011). El ancho de la fibra es de $47,4 \mu \mathrm{m}$, mayor que el que presentan otras fibras no maderables. Esto repre-

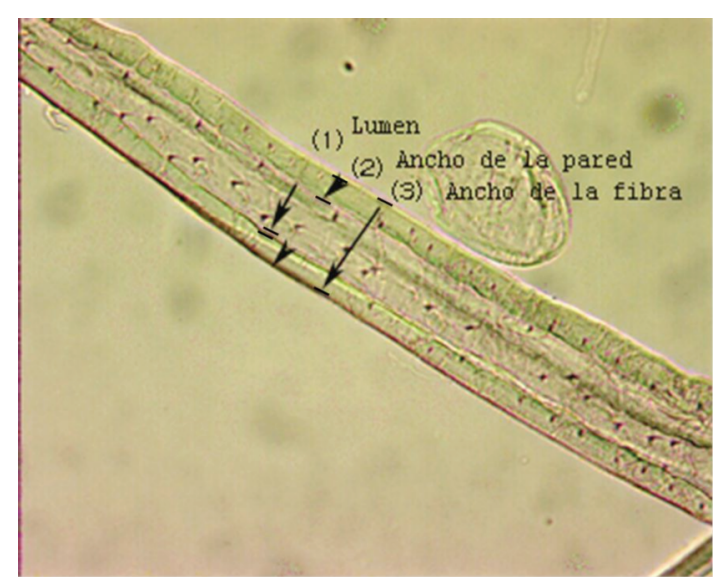

Figura 1. Propiedades biométricas de las fibras. 
senta una ventaja en el proceso de formación del papel, porque origina una rigidez mayor y aumenta considerablemente la resistencia al rasgado, así como una resistencia a la degradación por la acción mecánica durante el batido (Monteoliva, 2005; Núñez, 2004; Aguilar, 2011). Teniendo en cuenta el espesor de la pared y el ancho de la fibra, podemos decir que las fibras de mazorca presentan una pared delgada, de acuerdo con lo descrito por Petroff (1968). Las fibras de hojas de mazorca de maíz presentan un espesor de la pared celular promedio de $7,5 \mu \mathrm{m}$, característica que contribuye a la producción de un volumen mayor de la pulpa, hojas más gruesas y resistentes al rasgado, explosión y tensión, lo que queda comprobado con su coeficiente de flexibilidad (Tabla 3) y cuyo valor es mayor que 0,5 (Monteoliva, 2005). El lumen de estas fibras es, en promedio, de $32,1 \mu \mathrm{m}$, lo que hace aumentar la capacidad de impregnación en el proceso de pulpeo (Monteoliva, 2005).

El análisis de la fibra de bagazo de caña muestra una fibra con una longitud de $1,50 \mathrm{~mm}$, la cual, aun siendo mayor que la que presentan las maderas latifoliadas, no alcanza los valores observados en las coníferas. El ancho de fibra de 20 $\mu \mathrm{m}$, es menor que el observado en el caso de las hojas de maíz, pero le confiere al papel buena resistencia, sobre todo al rasgado. (Monteoliva, 2005; Núñez, 2004). La pared de las fibras se considera media, ya que la relación que hay entre el espesor y el ancho de fibra vale 0,4 (Petroff, 1968). Al igual que para el maíz, el lumen de esta fibra, $12,0 \mu \mathrm{m}$, origina un índice de flexibilidad de 0,6 (Tabla 3), lo que indica que el papel presentará buena resistencia a la tracción, por tener un mayor grosor de la hoja.

Comparando ambos materiales podemos afirmar que las fibras de las hojas de maíz presentan características morfológicas más adecuadas para la obtención de un papel con resistencia mecánica mayor, que el que se obtendría con las fibras de bagazo de caña. Existe un mayor colapso de fibras en el primer material y eso se refleja en una mejor calidad de la pulpa.

Índices de calidad de las fibras

A partir de la medición biométrica de las fibras: largo, ancho, lumen y espesor de la pared celular, se calcularon los índices de calidad de las pulpas, utili-

Tabla 2. Propiedades biométricas comparativas de fibras de diversas fuentes.

\begin{tabular}{lcccc}
\hline Especie & $\begin{array}{c}\text { Longitud promedio } \\
(\mathrm{mm})\end{array}$ & $\begin{array}{c}\text { Ancho de la fibra } \\
(\mu \mathrm{m})\end{array}$ & $\begin{array}{c}\text { Lumen } \\
(\mu \mathrm{m})\end{array}$ & $\begin{array}{c}\text { Espesor } \\
(\mu \mathrm{m})\end{array}$ \\
\hline Hoja de maíz & 1,86 & 47,4 & 32,1 & 7,5 \\
Bagazo de caña $_{\text {Eucalyptus globulus* }}$ & 1,50 & 20,0 & 12,0 & 4,0 \\
Kenaf $^{*}$ & 0,94 & 18,3 & 9,65 & 4,3 \\
Jacaranda acutifolia** $^{*}$ & 1,29 & 22,1 & 12,7 & 4,3 \\
Tilia mexicana** $_{\text {Bellotia mexicana** }}$ & 0,90 & 14,0 & 10,0 & 2,0 \\
Clethra mexicana** $^{*}$ & 1,31 & 28,0 & 20,0 & 4,0 \\
\hline
\end{tabular}

* Valores obtenidos para estas especies por Sanjuan (1997).

** Valores obtenidos para estas especies por Tamarit (1996). 
zando la relación de Runkel (Tabla 3). Cuanto menor sea, mayor flexibilidad de las fibras y mayor posibilidad de producir papel de buena calidad (Cea, 2011). Cuando este índice es menor que 0,25, se considera que la calidad de la pulpa es excelente para la fabricación de papel. Si el valor se sitúa entre 0,25 y 0,50, la calidad de la pulpa será muy buena. Entre 0,50 y 1,00 se tendrá una calidad buena. Valores entre 1,00 y 2,00 indican una calidad de pulpa regular y cuando el índice es mayor de 2,00, la calidad de la pulpa es mala (Larios, 1979).

La relación de Runkel de las fibras de hoja de mazorca las señala como adecuadas para producir un papel de muy buena calidad. Para el bagazo de caña, la calidad es un poco inferior, es decir, presenta un factor mayor, pero sigue siendo de calidad buena. Ambos materiales presentan mejores características generales para la obtención de pulpa que la mayoría de las plantas latifoliadas consideradas en la comparación.
Cuanto menor es la relación largo/ancho, mayor es la flexibilidad de las fibras y mayor la posibilidad de producir un papel de buena calidad. Cuando este factor es mayor que la unidad, la materia prima no es apropiada para dicha tarea (Santiago et al., 2002). El índice de flexibilidad indica que las fibras de hoja de mazorca tienen la propiedad de colapsarse parcialmente, por lo que se obtendrá una pulpa menos voluminosa. Además, presentan una sección transversal elíptica, una unión entre fibra y fibra buena, y la pared celular es delgada. Este coeficiente está relacionado directamente con la resistencia al doblez, a mayor flexibilidad, mayor capacidad de doblarse (Cea, 2011). El valor observado para el bagazo de caña es un poco inferior que el del maíz, por lo que su comportamiento diferirá un poco y su resistencia a ser doblado, será mayor.

El coeficiente de rigidez que presentan las fibras de hoja de mazorca es bajo, de acuerdo con la clasificación

Tabla 3. Índices de calidad de las fibras de las hojas de maíz y de bagazo de caña en comparación con otros materiales fibrosos.

\begin{tabular}{|c|c|c|c|c|}
\hline Tipo de fibra & $\begin{array}{l}\text { Relación } \\
\text { de Runkel }\end{array}$ & $\begin{array}{c}\text { Coeficiente } \\
\text { de rigidez }\end{array}$ & $\begin{array}{c}\text { Coeficiente } \\
\text { de flexibilidad }\end{array}$ & $\begin{array}{c}\text { Clasificación } \\
\text { calidad pulpa } \\
\text { según la relación } \\
\text { de Runkel }\end{array}$ \\
\hline \multicolumn{5}{|l|}{ Hoja de mazorca } \\
\hline Zea mays & 0,466 & 0,316 & 0,677 & Muy buena \\
\hline \multicolumn{5}{|l|}{ Bagazo de caña } \\
\hline Saccharum officinarum $\mathrm{L}$. & 0,660 & 0,400 & 0,600 & Buena \\
\hline Eucalyptus globulus* & 0,895 & 0,472 & 0,527 & Buena \\
\hline Eucalyptus dunnii* & 0,686 & 0,407 & 0,592 & Buena \\
\hline Jacaranda acutifolia** & 0,400 & 0,280 & 0,710 & Muy buena \\
\hline Tilia mexicana** & 0,400 & 0,280 & 0,710 & Muy buena \\
\hline Bellotia mexicana** & 0,510 & 0,340 & 0,650 & Buena \\
\hline Clethra mexicana** & 0,660 & 0,340 & 0,600 & Buena \\
\hline
\end{tabular}

*Valores obtenidos para estas especies por Sanjuan (1997).

** Valores obtenidos para estas especies por Tamarit (1996). 
presentada por Larios (1979) y cataloga a la pared de la fibra como delgada, por lo que la rigidez es baja. El bagazo de caña, sin embargo, presenta un valor superior, lo que implica que la pared de sus fibras celulares es de tipo medio, al igual que la rigidez. Este coeficiente influye de manera notoria en la resistencia al rasgado, siendo ésta una de las características más importantes en pulpas sin batir. Presenta además, una relación negativa en la porosidad de la hoja (Petroff, 1968).

De lo antes referido se desprende que la calidad de las fibras de las hojas de mazorca y del bagazo de caña son adecuadas para la producción de papel con características similares a las obtenidas con maderas de otras plantas anuales y plantas latifoliadas como el eucalipto. Sin embargo, se han considerado sólo las propiedades biométricas de las fibras, sin considerar la presencia del parénquima (células que no presentan carácter fibroso) cuyo aporte a las resistencias mecánicas del papel es bajo (Marques, 2008).

\section{Descripción de los elementos que conforman a la hoja de mazorca de maíz}

La planta de maíz está clasificada dentro del grupo de las monocotiledóneas, las cuales transportan las sales disueltas y los fotosintatos, desde las raíces hacia las hojas, y viceversa, a través de los vasos de xilema y floema (Fig. 2). Estos vasos, están reforzados por fibras, conformando, junto con tejido parenquimático, los haces fibrovasculares que se ordenan en nervaduras paralelinervas en toda la lámina. De estos haces fibrovasculares se obtienen las fibras que son materia prima para producir papel.

El tejido de las hojas de maíz tiene una estructura celular conformada por células parenquimatosas de paredes delgadas y con numerosas perforaciones (Fig. 3a) características en las gramíneas como el bagazo de caña de azúcar. Algunos de los vasos que se encuentran presentes son cribosos (vasos con puntuaciones, Fig. 3b) y otros espiralados (Fig. 3c).

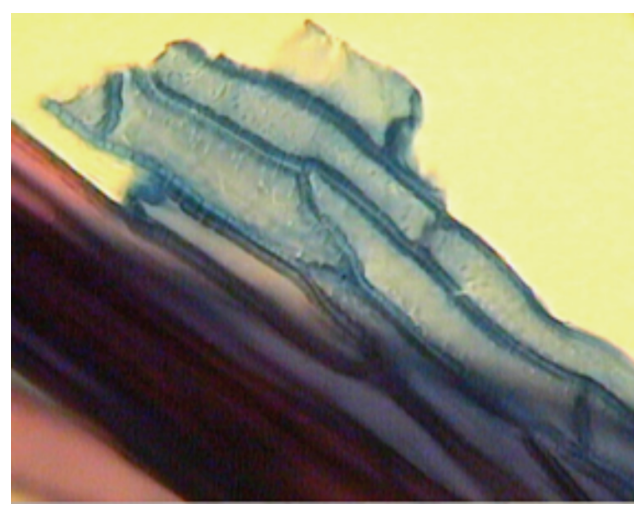

Figura 2. Segmentos de vasos y sistema del transporte de sustancias presentes en las hojas de mazorca de maíz. 

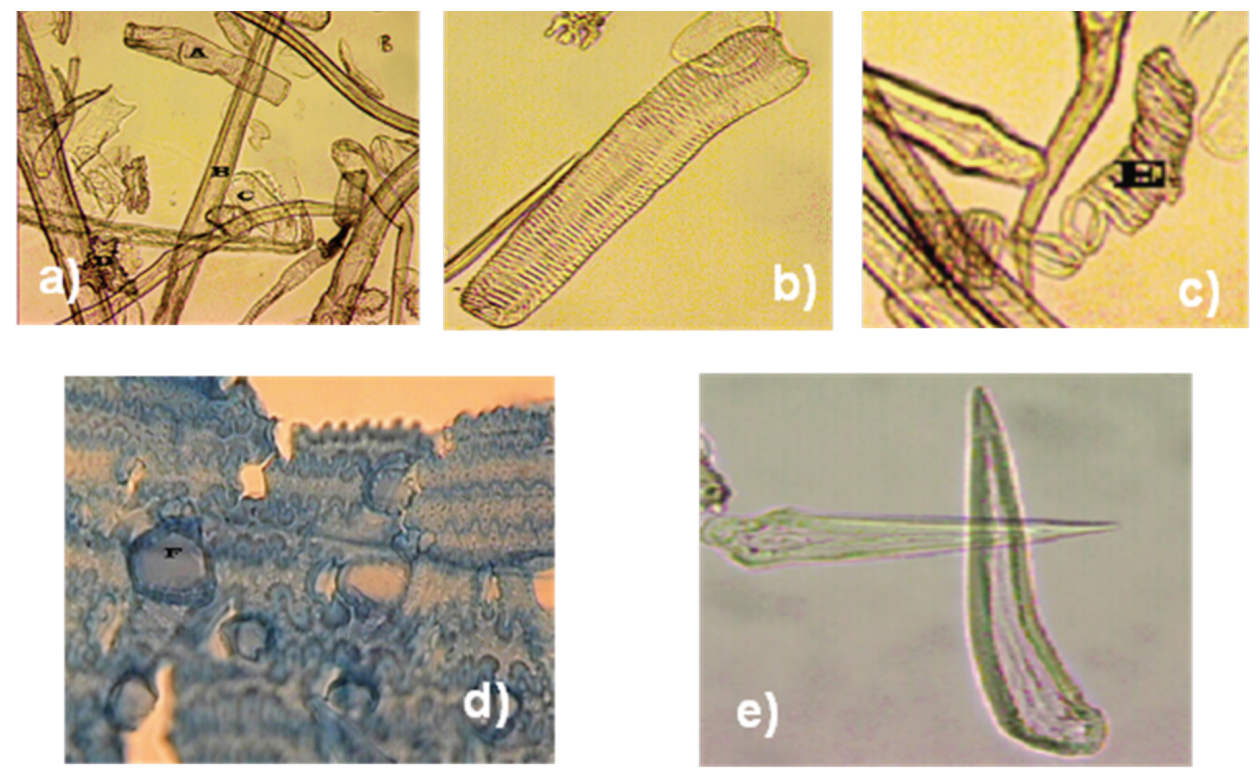

Figura 3. a) Elementos celulares. $A=$ Vaso criboso; $B=$ Fibra de pared gruesa; $\mathrm{C}=$ Parénquima; $\mathrm{D}=$ Células epidérmicas; b) Vaso criboso; c) $\mathrm{E}=$ Vaso espiralado;

d) Tejido epidérmico; e) Tricomas (pelos epidérmicos).

Las fibras son de pared celular gruesa, lumen medio, moderadamente largas y se caracterizan por presentar extremos romos, agudos, además de otras fibras dentadas y horquilladas con puntos en sus superficies. Las células epidérmicas o peines son rectangulares y presentan dientes en los bordes laterales (Fig. 3d). Otra característica morfológica de las hojas es la presencia de pelos epidérmicos o tricromas (Fig. $3 \mathrm{e}$ ).

El tejido del bagazo de caña tiene una estructura celular conformada por médula, compuesta principalmente por células parenquimatosas de paredes delgadas, elásticas y con numerosas perforaciones. Además de estar asociada a los haces fibrovasculares, los cuales son finos, de paredes delgadas con extremos romos, orquillas, sus superficies presentan ocasionalmente pequeños poros. Por otro lado, las fibras de la corteza son muy lignificadas, de mayor longitud, diámetro y paredes gruesas y presentan poros en toda la superficie (Stergios, 2006; Triana, 1990) (Fig. 4).

\section{CONCLUSIONES}

Por el alto contenido de holocelulosa y a-celulosa, tanto las hojas de mazorca de maíz, como el bagazo de caña, son en comparación con otras fuentes de fibra, materias primas adecuadas para la fabricación de papeles especiales y para la obtención de derivados de celulosa, situación que permite aprovechar el excedente de residuos.

El estudio biométrico de las fibras de las hojas de mazorca de maíz muestra que son un material fibroso con buenas propiedades de resistencia al batido. Además, poseen un lumen ancho, 


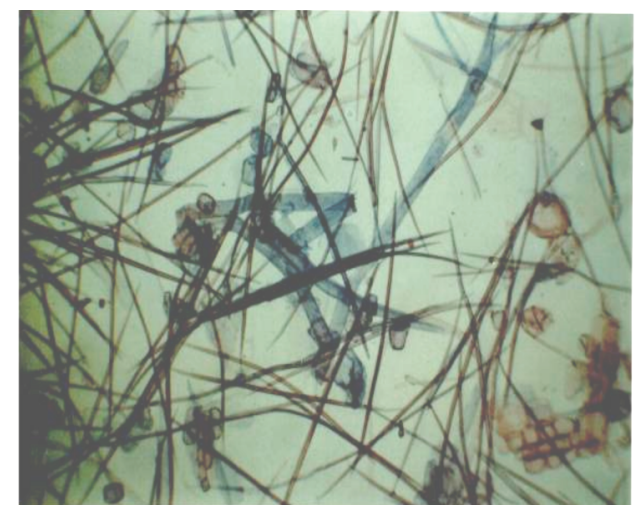

Figura 4. Morfología del bagazo de caña de azúcar.

aumentando con esto la capacidad de impregnación en el proceso de pulpeo. Los valores observados para el caso del bagazo de caña son menores, por lo que la rigidez, la resistencia al rasgado, a la explosión y a la tensión son inferiores. Aun así, la longitud de la fibra la sitúa dentro de las fibras largas, por lo que en su mezcla con las fibras de las hojas de mazorca de maíz, se obtiene una pulpa celulósica aprovechable.

Las propiedades biométricas de las fibras se ven afectadas por la presencia de una excesiva cantidad de parénquima en el material, quedando las propiedades de resistencia condicionadas a su comportamiento como material integral y su interacción con las fibras de la pulpa del bagazo de caña.

La hoja de maíz es una materia prima idónea para la obtención de papel especial, mezclada adecuadamente con bagazo de caña u otro material de fibra larga.

\section{AGRADECIMIENTOS}

Se agradece al Departamento de Madera Celulosa y Papel, del Centro Universitario de Ciencias Exactas e Ingenierías, de la
Universidad de Guadalajara, las facilidades prestadas para la utilización de los laboratorios de investigación del Centro y a los investigadores Jesús Rivera Prado, José Turrado, Alma Rosa y Salvador Pérez, por facilitar la utilización del Laboratorio de Tecnología del Papel y aportar asesoría en el laboratorio de pruebas físicas $\mathrm{Al}$ ingeniero Francisco Pérez Carrillo, de la empresa tamalera "La Candelaria", por el financiamiento privado que realizó para la ejecución de este trabajo.

\section{REFERENCIAS}

Aguilar, R.N., M.G. Galindo, M.J. Fortanelli, y S.C. Contreras. 2011. Factores de competitividad de la agroindustria de la caña de azúcar en México. Región y sociedad (52):261-297.

Aguilar, R.N. 2011. Efecto del almacenamiento de bagazo de caña en las propiedades físicas de celulosa grado papel. Ingeniería Investigación y Tecnología 12(1):189-197.

Akbari, M. y H. Resalati. 2012. Use of agricultural waste in the pulp and paper industry. 1th International and 4th 
National Congress on Recycling of Organic Waste in Agriculture. Irán. 4 p.

Casey, P.J. 1990. Pulpa y papel. Química y tecnología química. Volumen 1. Editorial Limusa. México. p:187-192.

Cea P.C.E. 2011. Evaluación de fibras celulósicas producidas en planta de celulosa Nueva Aldea. Tesis de maestría. Universidad Federal de Vicosa. Brasil. $156 \mathrm{p}$.

Conafor (Comisión Nacional Forestal). 1992. Ley Forestal Abrogada 2003. Ley General de Desarrollo Forestal Sustentable,www.conafor.gob.mx/portal/docs/ subsecciones/normateca/LGDFS.pdf. México DF, 74 p.

Conafor (Comisión Nacional Forestal). 2012. Programa nacional de dendroenergía forestal 2007-2012; http://www. conafor.gob.mx:8080/documentos/doc s/4/1585Programa\%20Nacional\%20D endroenergia.pdf. México DF. 12 p.

Estrada, M.M. 2010. Extracción y caracterización mecánica de las fibras de bambú (Guadua angustifolia) para su uso potencial como refuerzo de materiales compuestos. Tesis de maestría. Universidad de los Andes. Colombia. $146 \mathrm{p}$.

González, A.U. 1995. El maíz y su conservación. 1a. ed. Trillas. México. 399 p.

Hurter, W.R. 2001. Nonwood plant fiber uses in papermaking. Hurter Consult Incorporated. Extracted from "Agricultural Residues", Tappi. 1997. Non wood fibers short course notes, updated and expanded.

Imba, C.E.G. y C.L.M. Tallana. 2011. Aceptabilidad del bagazo de caña, rastrojo de maíz y tamo de cebada en bloques nutricionales como remplazo del maíz en cobayos de engorde (Cavia porcellus) en la granja la Pradera-Chaltura. Tesis. Universidad Técnica del Norte. Ibarra, Ecuador. 117 p.

IDEA (Instituto para la Diversificación y Ahorro de la Energía). 2007. Biomasa: Cultivos energéticos. Madrid. 56 p.

Juárez, M.M.A. 1993. Estudio anatómico de la madera de tres especies del estado de Jalisco. Tesis de licenciatura. Universidad de Guadalajara, Guadalajara. $75 \mathrm{p}$.

Larios, S.P. 1979. Índice de calidad de las pulpas de dos coníferas. Tesis profesional. Departamento de Bosques. Universidad Autónoma Chapingo. Texcoco, México. 68 p.

Matta, J.E. 1991. Papel periódico con bagazo y/o caña. II. Potencionalidad y manejo de las fibras. ATIPCA. 30(1):45-55.

Marques, S.G. 2008. Deslignificación de pasta Kraft de eucalipto con el polioxometalato SiW11 Mnlll en un proceso multietapas con peroxidasa versátil de Pleurotus eryngii. Universidad de Sevilla, España. 43 p.

Monteoliva, S. 2005. Propiedades de la madera de seis clones de Salix y su relación con el pulpado quimimecánico. Tesis doctoral. Universidad Nacional de La Plata, Facultad de Ciencias Agrarias y Forestales. Argentina. $219 \mathrm{p}$.

Núñez, C.E. 2004. Microestructura de la madera. PROCYP. Universidad Nacional de Misiones. Argentina. 5 p.

Palacios, J.H. 2003. Monografía de apoyo didáctico para el curso de estructuras anatómicas de materiales fibrosos para la maestría de productos fores- 
tales. Tesis de Licenciatura. Universidad de Guadalajara, Guadalajara. $175 \mathrm{p}$.

Pérez, M.M.A., H.R. Sánchez, L.D.J. Palma y G.S. Salgado. 2011. Caracterización química del compostaje de residuos de caña de azúcar en el sureste de México. Interciencia 36(1).

Petroff, G. y D. Normand. 1968. The correlation between the physical and mechanical properties of paper, and dimensional characteristics of papers, from tropical hardwoods. Pulp and paper development in Africa and the ear East. FAO I. 269-287.

Prado, M. 2007. Aprovechamiento de las hojas de maíz para la obtención de nuevas envolturas para tamales. Tesis de licenciatura. Universidad de Guadalajara, Guadalajara. 76 p.

López C., R. 2008. Productos forestales no maderables: importancia e impacto de su aprovechamiento, Revista Colombia Forestal (11):215-231

Ron, P.J., C.A. Jiménez, V.J. Carrera y L.J. Martín. 2006. Maíces nativos del occidente de México. Revista Científica Scientia 8(1):2-4.

Rutiaga, Q.J.G., H.J. Anzaldo, V.J.J. Vargas y D.R. Sanjuán. 2002. Propiedades de resistencia de una pulpa Kraft de pino mezclada con médula del bagazo de caña de azúcar. Madera y Bosques 8(2):17-26.

Sanjuán, D.R. 1997. Obtención de pulpas y propiedades de las fibras para papel. Editorial Universidad de Guadalajara, Guadalajara. 293 p.

Santiago, A.D.I., Rodríguez de G.N., G. Mogollón. 2002. Potencial papelero de la fibra de sisal (Agave sisalana).
Revista Forestal Venezolona 46(2): 19-27.

Saval, S. 2012. Aprovechamiento de residuos agroindustriales: pasado, presente y futuro, BioTecnología 16(2):14-46.

Sagarpa (Secretarçia de Agricultura, Ganadería, Desarrollo Rural, Pesca y Alimentación). 2010. Situación actual del azúcar, http://www.infoaserca. gob.mx/fichas/ficha33-Azucar 201009.pdf. México, D.F. 9 p.

Sener (Secretaría de Energía, Gobierno Federal). 2011. Iniciativa para el desarrollo de las energías renovables en México; http://sener.gob.mx/ webSener/res/0/D121122\%20Iniciativa\%20Renovable\%20SENER_Biom asa.pdf. México, DF.35p

Semarnat (Secretaría de Medio Ambiente y Recursos Naturales). 2011. (http://www.semarnat.gob.mx/temas/g estionambiental/forestalsuelos/Anuarios/ANUARIO_2011.pdf. México, D.F. $226 \mathrm{p}$.

Sidney, G.E. 1991. Mexican mills utilizing bagasse to produce pulp and fiberboard mill. Non-wood Plant Fiber Pulping 19:45-70.

Stergios, A. 2006. Identification of fiber components in packaging grades papers. IAWA Journal. 27(2):105-172.

Tamarit, U.J.C. 1996. Determinación de los índices de calidad de pulpa para papel de 132 maderas latifoliadas. Madera y Bosques 2(2):29-41.

Tappi Standards (Technical Association of the Pulp and Paper Industry). 1997. Asociación Técnica de la Industria de Pulpa y Papel de los Estados Unidos. Atlanta. 
Tapia-Tapia, E. del C., R. Reyes-Chilpa. 2008. Productos forestales no maderables en México: Aspectos económicos para el desarrollo sustentable. Madera y Bosques 14(3):95-112.

Triana. O. y M. Leonard, 1990, Atlas del bagazo de la caña de azúcar. Editorial Cuba 9. GEPLACEA. PNUD.ICIDICA. p:39-43.

Vega B.J., M.K. Delgado, B.M. Sibaja y A.P. Alvarado. 2008. Materiales poliméricos compuestos obtenidos a partir de los residuos generados por la agroindustria de la caña de azúcar. Una alternativa adicional. II. Revista iberoamericana de polímeros 9(4):389-406.

Wise, L.E., M. Marphy y A. d'Adieco. 1946. Chlorite holocellulose, its fractionation and bearing on summative wood analysis and studies on the hemicelluloses. Paper Trade Journal 122(2):35-43. ración de una pulpa celulósica mixta. Madera y Bosques 18(3):37-51. 